\title{
DEEP-FOCUS EARTHQUAKES IN THE MEDITERRANEAN REGION
}

\author{
by B. Gutenberg and C. F. Riuhter (")
}

Summury - In Table I the authors give a list of known deep-focus earthquakes in the eastern Mediterranean. Fig. 1 shows that their epicenters generally lie in the zones of active volcanoes, as in the regions surrounding the Pacific Ocean; however, their coincidence with positive gravity anomalies is less evident.

Zusc:mrienfnssung - In Tabelle I geber die Verff. eine Liste der ihnen bekannten Tiefenbeben im östlichen Mittelmeer. Fig. 1 zeigt, dags deren Ilerde im Allgemeinen in den Zonen der tätigen Vulkane liegen, ähnlich wie in der Umgebung des Pazifischen Ozeans. Dagegen ist deren '/usammenfallen mit positiven Schwereanomalien weniger deutlich.

Rinssunto - Gli AA. hanno raccolto nella Tab. 1 l'elenco dei terremoti profondi a loro noti del bacino mediterraneo orientale. La Fig. 1 mostra che in generalo gli epicentri relativi sono distribuiti nelle zone dei vulcani attivi, analogamente a quanto accade nelle regioni dell Oceano Pacifico. Per contro, la connessione con lo anomalie gravimetricho positive risulta meno evidente.

Certain of the larger earthquakes in the Levant are so abnormal in their effects that these were noticed and correctly interpreted by JuLIUs ScHMIDT as early as 1881. He concluded that these shocks must have a focal depth greater than that of ordinary earthquakes, and associated their occurrence in this region with the volcanic activity of Santorin and the Cyclades. Some of these intermediate shocks are the largest earthquakes in Europe. That of Aug. 11, 1903, in southern Greece was an intermediate shock of magnitude alcout 8 . A very large shock of this group (magnitude 7.9) occurred in 1926. It was investigated on the ground by SiEberg, who published a special study of the group (SiEBerg, 1932), which re termed Levantinische Riesenbeben; he did not consider the possibility of intermediate focal depth, but attempted an explanation in terms

(*) California Institute of Technology, Pasadena, California (U. S. A.). Balch Graduate School of the Geological Sciences: Contribution No. $45^{\mathrm{s}}$. 


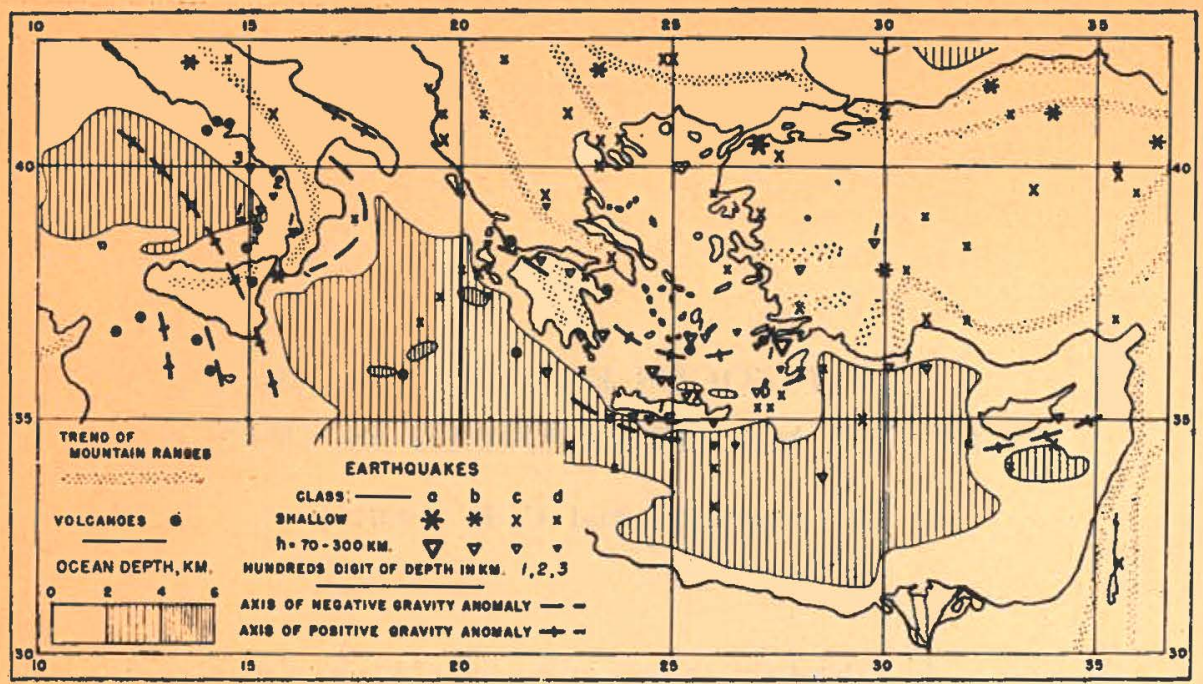

Fig. 1

of abnormal conduction of seismic energy along extended hypothetical lines of faulting. SiEBERG's figures show the onormous shaken area of some of these shocks, several of which were felt in Egypt, Oyrenaica, Asia Minor and far up the Adriatic.

Shocks at intermediate depth from 1903 to the present have now been

'I'ABLE I - Chronological list of deep-focus earihquales in the Mediterranean region

\begin{tabular}{|c|c|c|c|c|}
\hline Date & Time & Location & $\begin{array}{l}\text { Depth } \\
\text { in } \mathrm{km}\end{array}$ & Magnitudo \\
\hline 1908 , Nay $1 i$ & $12: 30.9$ & 35 N.. 24 E. & $100 \pm$ & $63 / 4$ \\
\hline 1910, February 18 & $05: 09.3$ & $36 \mathrm{~N} .24 \mathrm{l} / 2 \mathrm{IE}$. & $150 \pm$ & \\
\hline 1910, August 1 & $10: 40.4$ & 39 N. $15 \mathrm{E}$. & $200 \pm$ & $61 / 2$ \\
\hline 1910, A ugust 21 & $16: 11.5$ & 34 N. 27 E. & $170 \pm$ & $61 / 2$ \\
\hline 1911, April 4 & $15: 43.9$ & $361 / 2 \mathrm{~N} .251 / 2 \mathrm{E}$. & 140 & \\
\hline 1911, April 5 & $15: 28.2$ & 40 N. $151 / 2 \mathrm{E}$. & $200 \pm$ & $53 / 4$ \\
\hline 1911 , April 30 & $20: 42.5$ & $36 \mathrm{~N} .30 \mathrm{E}$. & 180 & $61 / 4$ \\
\hline 1915 , July 7 & $16: 42.9$ & 40 N. 15 E. & $300 \pm$ & \\
\hline 1918, July 16 & $20: 03: 46$ & $351 / 2 \mathrm{~N} .25 \mathrm{l} / 2 \mathrm{E}$. & $150 \pm$ & $61 / 2$ \\
\hline 1923, August 1 & $08: 16: 38$ & $35 \mathrm{~N} .25 \mathrm{E}$. & 150 & 6.7 \\
\hline 1925, July 6 & $12: 15: 55$ & $38 \mathrm{l} / 4 \mathrm{~N} \cdot 21 \mathrm{3} / 4 \mathrm{E}$. & 120 & $61 / 2$ \\
\hline 1926, June 26 & $19: 4 \beta: 34$ & $361 / 2 \mathrm{~N} .2 i 1 / 2 \mathrm{E}$. & 100 & 7.9 \\
\hline 1926, July 5 & $09: 21: 54$ & 36 I/2 N. $27 \mathrm{E}$. & $150 \pm$ & $51 / 2 \pm$ \\
\hline 1926, August 30 & $11: 38: 12$ & $363 / 4$ N. $231 / 4 \mathrm{E}$. & 100 & 7.0 \\
\hline 1926, August 17 & $01: 42: 53$ & 39 N. $143 / 4$ E. & $100 \pm$ & $53 / 4 \pm$ \\
\hline 1926, September 19 & $01: 03: 57$ & 36 N. 22 E. & 80 & $61 / 4$ \\
\hline 1927, June 5 & $02: 24: 58$ & $36 \mathrm{~N} .31 \mathrm{E}$. & 120 & $61 / 4$ \\
\hline 1927, July 1 & $08: 19: 04$ & $363 / 4 \mathrm{~N} .223 / 4 \mathrm{E}$. & 120 & 6.9 \\
\hline
\end{tabular}




\begin{tabular}{|c|c|c|c|c|}
\hline Date & Time & Location & $\begin{array}{l}\text { Deptlit } \\
\text { in } \mathrm{km}\end{array}$ & Magnitude \\
\hline 1928, March 7 & $10: 55: 12$ & 38 3/4 N. 16 E. & 120 & B \\
\hline 1929, March 27 & $07: 41: 46$ & $363 / 4 \mathrm{~N} .261 / 2 \mathrm{E}$. & $120 \pm$ & $53 / 4 \div$ \\
\hline 1930, February 14 & $18: 38: 20$ & $353 / 4 \mathrm{~N} .243 / 4 \mathrm{E}$. & 130 & $63 / 4$ \\
\hline $1930, \operatorname{March} 6$ & $08: 21: 42$ & $34 \mathrm{l} / 2 \mathrm{~N} .26 \mathrm{E}$ & 130 & $53 / 4$ \\
\hline 1930, March 6 & $09: 18: 32$ & $35 \mathrm{~N} .241 / 2 \mathrm{E}$. & 100 & 6 \\
\hline 1931, June 30 & $10: 23: 56$ & $361 / 2$ N. $23 \mathrm{E}$. & $100 \pm$ & $51 / 2 \pm$ \\
\hline 1932 , August 15 & $04: 34: 40$ & $39 \mathrm{l} / 4$ N. $22 \mathrm{E}$. & $100^{-}$ & $51 / 2$ \\
\hline 1933, July 19 & $20: 07: 08$ & $38 \mathrm{~J} / 4$ N. $293 / 4 \mathrm{E}$. & 100 & \\
\hline 1934, November 9 & $13: 40: 56$ & $363 / 4$ N. $253 / 4 \mathrm{E}$. & 140 & $61 / 4$ \\
\hline 1935, February 25 & $02: 51: 37$ & $353 / 4$ N. $25 \mathrm{E}$. & 80 & $63 / 4$ \\
\hline 1935, March 18 & $08: 40: 41$ & $351 / 2$ N. $27 \mathrm{E}$ & 130 & $61 / 4$ \\
\hline 1936, April 28 & $23: 15: 26$ & $363 / 4 \mathrm{~N} .263 / 4 \mathrm{E}$. & 170 & $53 / 4 \pm$ \\
\hline 1937, Decer & $17: 35: 30$ & 35 N. $231 / 2 \mathrm{E}$. & 100 & $61 / 2$ \\
\hline 1938, April 13 & $02: 45: 46$ & 39.2 N. 15.2 E. & 270 & $6.3 / 4$ \\
\hline 1938, June 3 & $16: 58: 03$ & $341 / 2 \mathrm{~N}, 261 / 2 \mathrm{E}$. & 120 & $5.3 / 4$ \\
\hline 1938, Septe & $03: 50: 38$ & 38 N. $221 / 2 \mathrm{E}$. & 100 & $61 / 2$ \\
\hline $1939, \mathrm{Se}$ & $00: 19: 31$ & 38 N. $20 \mathrm{l}$ & 80 & $61 / 2$ \\
\hline 1940, July 24 & $22: 15: 27$ & $341 / 2$ N. $34 \mathrm{E}$. & 80 & $53 / 4 \pm$ \\
\hline 1941, January 20 & $03: 37: 07$ & $35 \mathrm{~N} .34 \mathrm{E} \pm 2$ & $100 \pm 50$ & $61 / 2$ \\
\hline 1941, Marc & $16: 35: 15$ & $381 / 2$ N. $11 / / 2 \mathrm{E}$. & 100 & $61 / 2 \pm$ \\
\hline 1941, Marc & $18: 48: 21$ & $381 / 2 \mathrm{~N} .111 / 2 \mathrm{E}$. & 100 & $53 / 4 \pm$ \\
\hline 1941, December 13 & $06: 16: 05$ & 37 N. 28 E. & 100 & $6 \pm$ \\
\hline 1942, May 9 & $04: 37: 07$ & $351 / 2$ N. $26 \mathrm{E}$. & 100 & $53 / 4$ \\
\hline 1942, May 21 & $03: 42: 41$ & $37 \mathrm{~J} / 2 \mathrm{~N} .201 / 2 \mathrm{E}$. & 150 & $5 \mathrm{l} / 2 \pm$ \\
\hline 1942, June 21 & $04: 38: 43$ & $361 / 2$ N. $27 \mathrm{E}$. & 130 & $61 / 4$ \\
\hline 1943, Janu & $11: 14: 45$ & $381 / 2$ N. $201 / 2 \mathrm{E}$. & 100 & $51 / 2 \pm$ \\
\hline 1943, June 27 & $10: 05: 37$ & 35 N. 26 E. & 100 & $53 / 4 \pm$ \\
\hline 1943, September 17 & $03: 39: 20$ & $39 \mathrm{l} / 2 \mathrm{~N} .15 \mathrm{l} / 2 \mathrm{E}$. & $270 \pm$ & $51 / 2 \pm$ \\
\hline 1943 , October 16 & $13: 08: 53$ & $361 / 2$ N. $271 / 2 \mathrm{E}$. & 110 & $61 / 4 \pm$ \\
\hline 1944, May 27 & $23: 52: 30$ & $36 \mathrm{~N} .271 / 2 \mathrm{E}$. & 100 & $61 / 4$ \\
\hline 1944, August 9 & $17: 36: 37$ & $36 \mathrm{l} / 2 \mathrm{~N} .27 \mathrm{l} / 2 \mathrm{E}$. & 100 & $51 / 2 \pm$ \\
\hline 1945, Septomber 2 & $11 ; 53: 57$ & $333 / 4$ N. $281 / 2 \mathrm{E}$. & 80 & $6 \mathrm{I} / 2 \pm$ \\
\hline 1947, July 31 & $07: 54: 52$ & 39 l/4 N. 15 l/4 E. & 290 & $51 / 2 \pm$ \\
\hline 1947, September I & $22: 18: 54$ & $391 / 4$ N. $151 / 4 \mathrm{E}$. & $250 \pm$ & $51 / 2 \pm$ \\
\hline
\end{tabular}

identified. These were previously frequently overlooked, as most of the stations are at such distances that the usual evidence of depth is not available. Investigation of amplitudes of $P, S$, and surface waves has discriminated many of these shocks from the shallow earthquakes of the region.

Off the west coast of Italy several shocks are now known at depths approaching $300 \mathrm{~km}$, which makes them the deepest shocks identified outside the Pacific area. Shocks at depths of about $100 \mathrm{~km}$ off Sicily have been studied by DI FiLIPPo (1941).

Table I is a chronological list of all deep-focus shocks in the region for which data available to the writers suffice to determine epicenter and depth. These are plotted as triangles on Figure 1, which also shows gravity anomalies and active volcanoes. As in most regions, the epicenters of 
deepfocus earthquakes fall within the belts of contemporary volcanism, while shallow earthquakes (mapped as crosses and stars) generally fall outside these belts. Correlation of deep shocks and active volcanoes with positive gravity anomalies is much less definite than in the Pacific region. Elsewhere in Europe deep shocks are known only in Rumania.

Further details, and correlation between seismicity and other phenomena for the world, will be found in a forthcoming publication (GUTENBERG and RICHTER, 1948) from which the material of the present article is taken.

\section{REFERENCES}

Dr H'ILIPPo D., (1941) Studio microsismico del terremoto dul busso Tirreno del 16 Mrkrzo 1941, Soc. Sismol. Italiana, Boll. Vol. 39.

Gutanberg B., and Richter, C. F., (1948) Seismicity of the earth wind ts8ro. cinted phenomena. Princeton University Press (now in course of publication).

Schmidr, JuLIUs (1881), Studien über Vulkane und Erdbebcn, Leipzig.

SIEBERG A., (1932) Untersuchungen über Erdbeben und Bruchschollenbar im östlichen Mittelmeergebiet, Medizinisch-Naturwissenschaftliche Gesellschaft zu Jena, Denkschriften Band 18, Lieferung 2, pp. 161-273. 